\title{
Diseño e implementación del sistema MRP en las pymes
}

Juan Manuel Rivera Poma* Edith Ortega Pernia** Julio Pereyra Quiroz***

\section{RESUMEN}

El presente artículo aborda el tema de la Planeación de Requerimiento de Materiales (MRP) y su importancia como sistema administrativo de planificación y administración de los materiales requeridos para los procesos productivos de una empresa. El objetivo de esta investigación es presentar procedimientos adecuados y recomendaciones para la implementación del MRP en las pynes, mediante explicaciones simplificadas para lograr una implementación más exitosa. Finalmente, se presentan los beneficios obtenidos en un caso concreto y se llega a la conclusión de que el MRP, cuando es bien utilizado, proporciona una solución para las PYME en aspectos de control, reducción de costos de inventarios y abastecimiento oportuno.

Palabras clave: MRP, costos de almacenamiento, lead time, nivel de inventario, calidad, integración.

DESIGN AND IMPLEMENTATION OF MRP SYSTEM IN SMES

\section{ABSTRACT}

This article addresses the issue of Material Requirements Planning (MRP) and its importance as an administrative system planning and management of the materials required for the production processes of a company. The objective of this research is to present proper procedures and recommendations for the implementation of MRP in SMEs through simplified explanations for successful implementation. Finally, the benefits obtained in a particular case and conclude that the MRP, when properly used are presented, provides a solution for SMEs in areas such as minimizing inventory costs and supply at the required time.

Keywords: MRP, storage costs, lead time, inventory level, quality, integration

\section{INTRODUCCIÓN}

En el mercado, cada vez más competitivo, las empresas en crecimiento suelen enfrentar frecuentemente problemas de inventarios excesivos, pedidos entregados con retraso, altos costos de producción y calidad inconsistente. Lo que genera pérdidas económicas y de participación en el mercado. El sistema de Planeamiento de requerimientos de Materiales (MRP) ${ }^{1}$ como complemento ideal para la planeación y control de la producción tiene grandes éxitos en las empresas grandes como una herramienta fundamental para asegurar el abastecimiento del material oportuno, disminuir los costos de almacenamiento y evitar la paralización de la producción por falta de material.*

En el contexto actual, la calidad de los bienes y servicios ofrecidos es un factor fundamental, lo que significa mejorar los procesos que forman parte de la cadena de valor de la empresa, MRP proporciona soluciones para lograr este propósito y con una implementación adecuada puede llegar a convertirse en fuente de ventaja competitiva y sostenible en el tiempo.

Debemos entender que el proceso de implementación no es un hecho aislado, requiere de la participación e integración de todas las áreas de la empresa y de su compromiso en todas sus acciones.

\section{PLANEACIÓN DE REQUERIMIENTO DE MATERIALES (MRP)}

Es una metodología que requiere conocer la demanda independiente de los productos finales de la empresa para calcular de forma rápida y precisa la demanda dependiente generada por el requerimiento de los productos. MRP también nos proporciona un programa para producir o pedir la materia prima.

El sistema MRP requiere de información, tanto del proceso productivo como de la demanda de los productos, por lo que se utiliza un software especializado para el procesamiento de la información. La fiabilidad del sistema MRP dependerá exclusivamente de la fiabilidad de los datos proporcionados.

El sistema MRP necesita los siguientes datos de entrada:

\footnotetext{
Magíster en Ing. Industrial, Docente en la Universidad Nacional Mayor de San Marcos (UNMSM). E-mail: jmriverap@yahoo.es

** Estudiante de Ingeniería Industrial UNMSM. E-mail: edith.ortega@industrial.unmsm.pe

1 Material Requirement Planning = MRP, por sus siglas en inglés.
} 


\section{A. Plan maestro de producción (MPS) ${ }^{2}$}

EI MPS se construye a partir de los pedidos de los clientes o de pronósticos de la demanda, identifica las cantidades de cada uno de los productos terminados y determina en qué periodos, dentro del horizonte de planeación, es necesario producir.

\section{B. Lista de materiales (BOM) ${ }^{3}$}

Identifica la estructura de los productos terminados, especificando los siguientes aspectos:

Artículos subcomponentes, así como su cantidad requerida en cada nivel.

Secuencia de integración o ensamble de los artículos subcomponentes.

Centros de trabajo en los que se realizan los ensambles.

\section{Registros de inventario}

El sistema de la empresa debe contener un archivo de registros de inventario actualizado para cada uno de los artículos en la estructura de los productos. Se debe contar con los siguientes datos:

- Identificación de los artículos mediante códigos.

- Cantidad disponible.

- Nivel de stock de seguridad.

- Tiempo de abastecimiento de artículos (lead time).

\section{FACTORES CRÍTICOS DE ÉXITO EN LA IM- PLEMENTACIÓN DE UN SISTEMA DE GESTIÓN EMPRESARIAL}

La implementación de una solución MRP puede ser un éxito tecnológico pero un fracaso organizacional a causa de deficiencias en el proceso social y político del desarrollo del proyecto. Se debe prestar suficiente importancia a la participación, liderazgo y compromiso de los miembros claves de la empresa en cada una de las etapas de la implementación.

Se destacan los siguientes cuatro factores para lograr el éxito en una implementación de un sistema MRP:

\subsection{Definición del objetivo}

Definir qué es lo que se quiere lograr y comunicarlo explícitamente a toda la organización. Los objetivos

MPS $=$ Master Production Schedule

$\mathrm{BOM}=$ Bill of Materials deben ser claros, específicos y deben indicar la dirección general del proyecto.

En la etapa de análisis inicial y planeamiento es donde se definen los objetivos, por tanto es considerada por muchos expertos como la etapa más importante del proyecto, ya que de esta etapa depende el cumplimiento de las siguientes etapas.

3.2. Participación, compromiso y liderazgo de los involucrados en la implementación del sistema

Lo cual implica contar con el apoyo total de la Gerencia, con un líder de proyecto comprometido y con usuarios involucrados que se comprometan con el nuevo sistema, capacitándose apropiadamente.

\subsection{El método de trabajo}

Se debe elaborar y cumplir con un método sistemático de trabajo, comprensible para todos, con objetivos claros, control de avance del proyecto, asignación de responsabilidades y roles, cumplimiento de compromisos, asignación de recursos, generación de documentos de control, seguimiento del proyecto y una planificación detallada de las actividades de cada una de las etapasde la metodología de implementación.

\subsection{La idoneidad de la solución de sistema MRP}

El sistema debe cumplir con las características básicas, de ser flexible y adaptarse a las exigencias de la empresa, permitiéndole crecer hacia nuevos planteamientos de negocio.

\section{PROCEDIMIENTO DE IMPLEMENTACIÓN}

A continuación presentamos un método práctico de organizar los datos de entrada necesarios para el MRP, e ingresarlos posteriormente al software determinado.El caso desarrollado es de una empresa fabricante de empaques de cartón corrugado.

\section{Plan maestro de producción}

La empresa, de estudio, maneja su plan de producción en base a la demanda recurrente de años anteriores.

En la siguiente tabla, se muestra elresumen del plan maestro de producción para el periodo de estudio. 
Tabla 4.1. Resumen del Plan maestro de producción

\begin{tabular}{|c|c|c|}
\hline \multicolumn{3}{|c|}{$\begin{array}{l}\text { MPS de una empresa que elabora empaques de } \\
\text { cartón }\end{array}$} \\
\hline Meses & $\begin{array}{l}\text { Láminas de } \\
\text { cartón }\end{array}$ & $\begin{array}{l}\text { Total días } \\
\text { laborados }\end{array}$ \\
\hline Febrero & 3.960 .601 & 20 \\
\hline Marzo & 4.853 .221 & 20 \\
\hline Abril & 3.984 .055 & 20 \\
\hline Mayo & 4.089 .312 & 20 \\
\hline $\begin{array}{l}\text { Total de } \\
\text { productos }\end{array}$ & $\begin{array}{c}\text { 16.887.189 } \\
\text { Láminas }\end{array}$ & \\
\hline
\end{tabular}

Fuente: Elaboración propia.

Tabla 4.2. Detalle del Plan Maestro de Producción

\begin{tabular}{|c|c|c|c|c|}
\hline \multirow[b]{2}{*}{$\begin{array}{c}\text { Días } \\
\text { laborados }\end{array}$} & \multicolumn{4}{|c|}{ Producción de "Lámina" } \\
\hline & Febrero & Marzo & Abril & Mayo \\
\hline Día 1 & 193.277 & 194.129 & 196.016 & 191.789 \\
\hline Día 2 & 196.446 & 242.176 & 197.609 & 172.160 \\
\hline Día 3 & 198.426 & 243.146 & 197.211 & 204.875 \\
\hline Día 4 & 192.485 & 235.867 & 199.203 & 198.332 \\
\hline Día 5 & 200.010 & 245.088 & 220.717 & 226.548 \\
\hline Día 6 & 237.636 & 290.223 & 194.422 & 202.421 \\
\hline Dia 7 & 204.763 & 250.912 & 199.203 & 211.417 \\
\hline Día 8 & 196.05 & 240.234 & 200.796 & 202.421 \\
\hline Día 9 & 189.713 & 232.469 & 190.836 & 245.359 \\
\hline Día 10 & 200.010 & 245.088 & 201.195 & 203.239 \\
\hline Día 11 & 199.614 & 240.234 & 197.211 & 202.421 \\
\hline Día 12 & 198.030 & 236.837 & 199.601 & 199.149 \\
\hline Día 13 & 185.752 & 242.661 & 199.203 & 199.967 \\
\hline Día 14 & 193.277 & 249.941 & 187.251 & 201.194 \\
\hline Día 15 & 199.218 & 236.837 & 199.203 & 202.012 \\
\hline Día 16 & 192.881 & 244.117 & 200.398 & 200.376 \\
\hline Día 17 & 197.238 & 239.749 & 194.023 & 199.149 \\
\hline Día 18 & 197.238 & 241.690 & 198.406 & 203.648 \\
\hline Día 19 & 197.634 & 242.176 & 198.804 & 204.057 \\
\hline Día 20 & 192.089 & 235.381 & 193.227 & 198.332 \\
\hline $\begin{array}{c}\text { Total } \\
\text { producción }\end{array}$ & 3.960 .601 & 4.853.221 & 3.984 .055 & 4.089.312 \\
\hline
\end{tabular}

Fuente: Elaboración propia.

\section{Lista de materiales (BOM)}

La lista de materiales al igual que el inventario es elaborado en Excel, en forma de tablas, para este estudio se elaborará el BOM en forma de árbol, con el objetivo de visualizar cada uno de los componentes que se requieren para elaborar un empaque de cartón corrugado.

\section{Inventario}

El inventario de materia prima se refleja la cantidad de materia prima que tiene en existencia física, son valores que se reflejan en su base de datos durante el periodo de estudio, por mes y de cada insumo a utilizar.

Tabla 4.3. Inventario de cajas

\begin{tabular}{|c|c|c|}
\hline Cajas & $\begin{array}{c}\text { Inventario Inicial } \\
\text { 01 “Laminas" }\end{array}$ & $\begin{array}{c}\text { Inventario Final } \\
\text { 30 “Láminas" }\end{array}$ \\
\hline Febrero & 225.312 & 228.935 \\
\hline Marzo & 228.935 & 200.232 \\
\hline Abril & 200.232 & 208.965 \\
\hline Mayo & 208.965 & 190.268 \\
\hline
\end{tabular}

Fuente: Elaboración propia

Tabla 4.4. Inventario de tinta

\begin{tabular}{|c|c|c|}
\hline Tinta & $\begin{array}{c}\text { Inventario Inicial } \\
\text { 01 “Kilos" }\end{array}$ & $\begin{array}{c}\text { Inventario Final } \\
\text { 30 “Kilos" }\end{array}$ \\
\hline Febrero & 5.000 & 9.000 \\
\hline Marzo & 9.000 & 6.100 \\
\hline Abril & 6.100 & 4.250 \\
\hline Mayo & 4.250 & 9.530 \\
\hline
\end{tabular}

Fuente: Elaboración propia.

Tabla 4.5. Inventario de adhesivos

\begin{tabular}{|c|c|c|}
\hline Adhesivos & $\begin{array}{c}\text { Inventario Inicial } \\
\text { 01 "Kilos" }\end{array}$ & $\begin{array}{c}\text { Inventario Final } \\
\text { 30 “Kilos" }\end{array}$ \\
\hline Febrero & 2.250 & 2.469 \\
\hline Marzo & 2.469 & 3.000 \\
\hline Abril & 3.000 & 1.580 \\
\hline Mayo & 1.580 & 8.126 \\
\hline
\end{tabular}

Fuente: Elaboración propia.

Tabla 4.6. Inventario de piola

\begin{tabular}{|c|c|c|}
\hline Piola & $\begin{array}{c}\text { Inventario Inicial } \\
\text { 01 "Kilos" }\end{array}$ & $\begin{array}{c}\text { Inventario Final } \\
\text { 30 “Kilos" }\end{array}$ \\
\hline Febrero & 110 & 125 \\
\hline Marzo & 125 & 135 \\
\hline Abril & 135 & 110 \\
\hline Mayo & 110 & 135 \\
\hline
\end{tabular}

Fuente: Elaboración propia. 
Figura 4.1. Lista de materiales

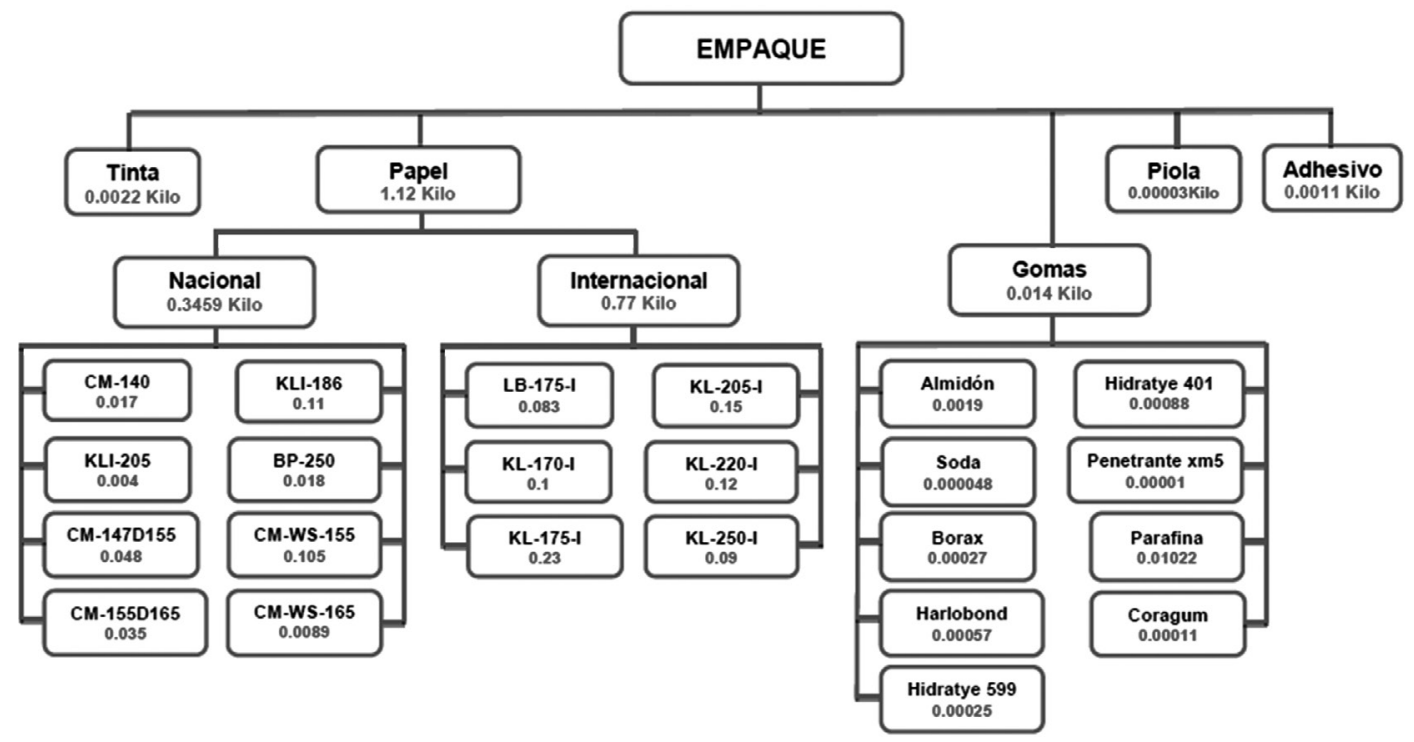

Fuente: Elaboración propia.

Tabla 4.7. Inventarios de papel (feb)

\begin{tabular}{|c|c|c|c|}
\hline & PAPEL & $\begin{array}{l}\text { Inventario } \\
\text { inicial } \\
\text { 01-Febrero } \\
\text { "Kilos" }\end{array}$ & $\begin{array}{c}\text { Inventario } \\
\text { final } \\
\text { 30-Febrero } \\
\text { "Kilos" }\end{array}$ \\
\hline \multirow{7}{*}{$\begin{array}{l}\bar{\pi} \\
\frac{0}{0} \\
\frac{0}{0} \\
z\end{array}$} & KLI-186 & 245.00 & 325.000 \\
\hline & KLI-205 & 10.500 & 13.500 \\
\hline & CM-147D155 & 115.000 & 125.000 \\
\hline & CM-155D165 & 85.000 & 158.000 \\
\hline & CM-140 & 35.000 & 35.000 \\
\hline & BP-250 & 40.125 & 50.125 \\
\hline & CM-WS-165 & 242.000 & 270.450 \\
\hline \multirow{6}{*}{ 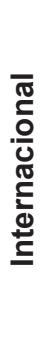 } & LB-175-I & 410.000 & 550.283 \\
\hline & KL-170-I & 638.000 & 787.300 \\
\hline & KL-175-I & 950.500 & 1.250 .000 \\
\hline & KL-205-I & 715.000 & 700.600 \\
\hline & KL-220-I & 605.500 & 620.730 \\
\hline & KL-250-I & 522.000 & 558.316 \\
\hline
\end{tabular}

Fuente: Elaboración propia.
Tabla 4.8. Inventarios de papel (mar)

\begin{tabular}{|c|c|c|c|}
\hline & PAPEL & $\begin{array}{c}\text { Inventario } \\
\text { inicial } \\
\text { 01-Marzo } \\
\text { "Kilos" }\end{array}$ & $\begin{array}{c}\text { Inventario } \\
\text { final } \\
\text { 30-Marzo } \\
\text { "Kilos" }\end{array}$ \\
\hline \multirow{7}{*}{$\begin{array}{l}\bar{\pi} \\
\frac{0}{5} \\
\frac{0}{0} \\
\frac{\pi}{Z}\end{array}$} & KLI-186 & 325.000 & 250.000 \\
\hline & KLI-205 & 13.500 & 19.500 \\
\hline & CM-147D155 & 125.000 & 115.000 \\
\hline & CM-155D165 & 158.000 & 80.000 \\
\hline & CM-140 & 35.000 & 45.000 \\
\hline & BP-250 & 50.125 & 45.000 \\
\hline & CM-WS-165 & 350.000 & 200.000 \\
\hline \multirow{6}{*}{ 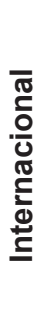 } & LB-175-I & 550.283 & 450.000 \\
\hline & KL-170-I & 787.300 & 571.727 \\
\hline & KL-175-I & 1.250 .000 & 1.200 .000 \\
\hline & KL-205-I & 700.600 & 612.612 \\
\hline & KL-220-I & 620.730 & 500.000 \\
\hline & KL-250-I & 558.316 & 450.000 \\
\hline
\end{tabular}

Fuente: Elaboración propia. 
Tabla 4.9. Inventarios de papel (abril)

\begin{tabular}{|c|c|c|c|}
\hline & PAPEL & $\begin{array}{l}\text { Inventario } \\
\text { inicial } \\
\text { 01-Abril } \\
\text { "Kilos" }\end{array}$ & $\begin{array}{c}\text { Inventario } \\
\text { final } \\
\text { 30-Abril } \\
\text { "Kilos" }\end{array}$ \\
\hline \multirow{7}{*}{$\begin{array}{l}\bar{\pi} \\
\frac{0}{0} \\
\frac{0}{0} \\
z\end{array}$} & CM140 & 45.000 & 45.840 \\
\hline & KLI186 & 250.000 & 225.000 \\
\hline & KLI205 & 19.500 & 10.500 \\
\hline & CM-155D165 & 80.000 & 90.220 \\
\hline & CM-147D155 & 115.000 & 115.054 \\
\hline & BP-250 & 45.000 & 42.121 \\
\hline & CM-WS-165 & 290.470 & 240.000 \\
\hline \multirow{6}{*}{ 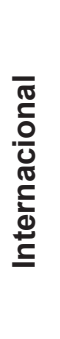 } & LB-175-I & 450.000 & 400.000 \\
\hline & KL-170-I & 571.727 & 571.727 \\
\hline & KL-175-I & 1.200 .000 & 983.522 \\
\hline & KL-205-I & 612.612 & 612.612 \\
\hline & KL-220-I & 500.000 & 598.320 \\
\hline & KL-250-I & 450.000 & 425.056 \\
\hline
\end{tabular}

Fuente: Elaboración propia.
Tabla 4.10. Invent. de papel (mayo)

\begin{tabular}{|c|c|c|c|}
\hline & PAPEL & $\begin{array}{c}\text { Inventario } \\
\text { inicial } \\
\text { 01-Mayo } \\
\text { "Kilos" }\end{array}$ & $\begin{array}{c}\text { Inventario } \\
\text { final } \\
\text { 30-Mayo } \\
\text { "Kilos" }\end{array}$ \\
\hline \multirow{7}{*}{$\begin{array}{l}\bar{\pi} \\
\frac{0}{0} \\
\frac{0}{0} \\
z\end{array}$} & CM140 & 45.840 & 43.340 \\
\hline & KLI186 & 225.000 & 200.000 \\
\hline & KLI205 & 10.500 & 11.250 \\
\hline & CM-155D165 & 90.220 & 75.860 \\
\hline & CM-147D155 & 115.054 & 135.220 \\
\hline & BP-250 & 42.121 & 48.000 \\
\hline & CM-WS-165 & 225.650 & 198.300 \\
\hline \multirow{6}{*}{ 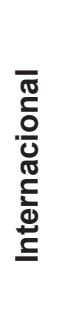 } & LB-175-I & 400.000 & 350.000 \\
\hline & KL-170-I & 571.727 & 585.000 \\
\hline & KL-175-I & 983.522 & 102.000 \\
\hline & KL-205-I & 612.612 & 625.300 \\
\hline & KL-220-I & 598.320 & 575.600 \\
\hline & KL-250-I & 425.056 & 450.256 \\
\hline
\end{tabular}

Fuente: Elaboración propia.

Tabla 4.11. Inventario de goma

\begin{tabular}{|c|c|c|c|c|c|c|c|c|c|c|}
\hline & & Almidon & Soda & Borax & Harlobond & $\begin{array}{c}\text { Hidratye } \\
599\end{array}$ & $\begin{array}{c}\text { Hidratye } \\
401\end{array}$ & $\begin{array}{c}\text { Penetrante } \\
\text { XM5 }\end{array}$ & Parafina & $\begin{array}{l}\text { Coragum } \\
\text {-R55 }\end{array}$ \\
\hline \multirow{4}{*}{ 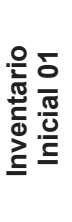 } & Febrero & 9.505 .00 & 226,7 & 165,30 & $1.156,68$ & 325,50 & 400,20 & 157,37 & $5.500,00$ & 150,25 \\
\hline & Marzo & 11.065 .00 & 235,3 & 166,00 & $1.179,36$ & 258,60 & 526,80 & 116,20 & $7.140,00$ & 175,00 \\
\hline & Abril & 9.700 .00 & 223,00 & 141,6 & $1.111,32$ & 226,80 & 396,80 & 100,00 & $6.260,00$ & 175,00 \\
\hline & Mayo & 7.505 .00 & 289,5 & 147,5 & $1.111,32$ & 226,80 & 400,40 & 121,6 & $6.455,00$ & 454,5 \\
\hline \multirow{4}{*}{ 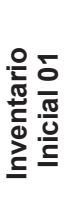 } & Febrero & 11.065 .00 & 235,3 & 166,00 & $1.179,36$ & 258,60 & 526,80 & 116,20 & $7.140,00$ & 175,00 \\
\hline & Marzo & 9.700 .00 & 223,00 & 141,55 & $1.111,32$ & 226,80 & 396,80 & 100,00 & $6.260,00$ & 454,54 \\
\hline & Abril & 7.505 .00 & 289,5 & 147,45 & $1.111,32$ & 226,80 & 400,40 & 121,61 & $6.455,00$ & 154,54 \\
\hline & Mayo & 9.996 .00 & 239,6 & 179,10 & 111,32 & 226,80 & 905,50 & 174,76 & $8.080,00$ & 454,54 \\
\hline
\end{tabular}

Fuente: Elaboración propia

\section{Lead time}

La empresa maneja el "lead time" de los ítems de la materia primaa base del tiempo establecido por sus proveedores para cumplir con la entrega de cada uno de los pedidos, Esta acción es importante para planificar el tiempo de reabastecimiento.

\section{Tamaño de lote}

La empresa determina el tamaño de lote dependiendo de la capacidad promedio de producción deempaques de cartón corrugado. En lo referente a los ítems de la materia prima considera el tiempo de elaboración de sus proveedores. 
Tabla 4.12. Lead time

\begin{tabular}{|c|c|c|c|c|c|}
\hline & & \multicolumn{4}{|c|}{ Lead time "Días" } \\
\hline & & Febrero & Marzo & Abril & Mayo \\
\hline & cajas & 1 & 1 & 1 & 1 \\
\hline \multirow{8}{*}{ 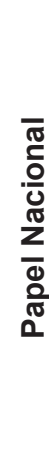 } & CM140 & 7 & 7 & 7 & 7 \\
\hline & KLI186 & 7 & 7 & 7 & 7 \\
\hline & KLI205 & 7 & 7 & 7 & 7 \\
\hline & BP250 & 7 & 7 & 7 & 7 \\
\hline & CM147D & 7 & 7 & 7 & 7 \\
\hline & CMWS165 & 7 & 7 & 7 & 7 \\
\hline & CMW155 & 7 & 7 & 7 & 7 \\
\hline & CM155D & 7 & 7 & 7 & 7 \\
\hline \multirow{6}{*}{ 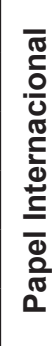 } & LB175I & 90 & 90 & 90 & 90 \\
\hline & KL205I & 90 & 90 & 90 & 90 \\
\hline & KL175I & 90 & 90 & 90 & 90 \\
\hline & KL170I & 90 & 90 & 90 & 90 \\
\hline & KL250I & 90 & 90 & 90 & 90 \\
\hline & KL220I & 90 & 90 & 90 & 90 \\
\hline \multirow{12}{*}{ 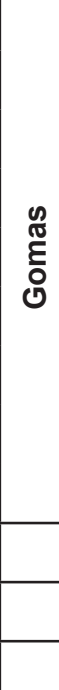 } & Almidón & 3 & 3 & 3 & 3 \\
\hline & Soda & 1 & 1 & 1 & 1 \\
\hline & Borax & 1 & 1 & 1 & 1 \\
\hline & Harlobond & 3 & 3 & 3 & 3 \\
\hline & Hidratye599 & 3 & 3 & 3 & 3 \\
\hline & Hidratye 401 & 1 & 1 & 1 & 1 \\
\hline & Penetrante & 1 & 1 & 1 & 1 \\
\hline & Parafina & 1 & 1 & 1 & 1 \\
\hline & Coragum-R55 & 3 & 3 & 3 & 3 \\
\hline & Adhesivos & 3 & 3 & 3 & 3 \\
\hline & Tinta & 3 & 3 & 3 & 3 \\
\hline & Piola & 3 & 3 & 3 & 3 \\
\hline
\end{tabular}

Fuente: Elaboración propia.

Con estos datos se logra proporcionar,en forma práctica,los datos al software MRP especializado.

\section{SOFTWARES}

En la actualidad muchas empresas que desarrollan sistemas computacionales se encuentran en
Tabla 4.13. Tamaño de lote

\begin{tabular}{|c|c|c|c|c|c|}
\hline & & Febrero & Marzo & Abril & Mayo \\
\hline & Cajas "Láminas" & 250.000 & 250.000 & 250.000 & 250.000 \\
\hline \multirow{8}{*}{ 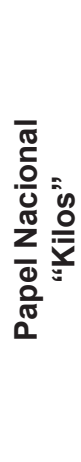 } & CM140 & 55.000 & 55.000 & 55.000 & 55.000 \\
\hline & KLI186 & 275.000 & 355.000 & 275.000 & 275.000 \\
\hline & KLI205 & 15.200 & 15.200 & 20.000 & 15.200 \\
\hline & BP250 & 50.000 & 55.000 & 50.000 & 50.000 \\
\hline & CM147D & 125.000 & 135.000 & 125.000 & 125.000 \\
\hline & CMWS165 & 300.000 & 300.000 & 300.000 & 100.000 \\
\hline & CMW155 & 250.000 & 450.000 & 250.000 & 250.000 \\
\hline & CM155D & 100.000 & 110.000 & 100.000 & 100.000 \\
\hline \multirow{6}{*}{ 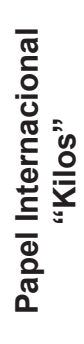 } & LB175I & 440.000 & 575.000 & 500.000 & 440.000 \\
\hline & KL205I & 800.000 & 800.000 & 750.000 & 800.000 \\
\hline & KL175। & 1.000 .000 & 1.300 .000 & 1.300 .000 & 1.000 .000 \\
\hline & KL170I & 650.000 & 800.000 & 650.000 & 650.000 \\
\hline & KL250I & 550.000 & 650.000 & 550.000 & 550.000 \\
\hline & KL220I & 625.000 & 625.000 & 625.000 & 625.000 \\
\hline \multirow{12}{*}{ 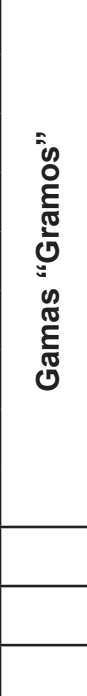 } & Almidón & 10.200 & 11.500 & 10.200 & 10.200 \\
\hline & Soda & 400 & 400 & 400 & 400 \\
\hline & Borax & 175 & 175 & 175 & 175 \\
\hline & Harlobond & 1.500 & 1.500 & 1.500 & 1.500 \\
\hline & Hidratye599 & 400 & 400 & 400 & 400 \\
\hline & Hidratye401 & 450 & 535 & 450 & 450 \\
\hline & Penetrante & 200 & 200 & 200 & 200 \\
\hline & Parafina & 6.500 & 7.500 & 6.500 & 6.500 \\
\hline & Coragum-R55 & 225 & 225 & 225 & 225 \\
\hline & Adhesivos "Kilos" & 3.500 & 3.500 & 3.500 & 3.500 \\
\hline & Tinta "Kilos" & 6.500 & 6.500 & 6.500 & 5.900 \\
\hline & Piola "Kilos" & 150 & 150 & 150 & 150 \\
\hline
\end{tabular}

Fuente: Elaboración propia. constante cambio y creando nuevos sistemas que faciliten mejorar los procesos. por ello desarrollan softwares MRP para llevar un control más automatizado a continuación se verá algunos de ellos sin dejar de analizar el sistema SAP que es uno de los más conocidos sistemas de información que gestiona de manera integrada todas las áreas funcionales de la empresa. 


\section{1. $\mathrm{SAP} \circledast$ Business One}

Diseñada específicamente para las pequeñas empresas, constituye una solución rentable para gestionar toda la empresa con mayor claridad, desde las finanzas hasta el inventario, pasando por las ventas y las relaciones con los clientes. Ayuda a optimizar las operaciones globales, tener acceso instantáneo a información completay acelerar el crecimiento rentable.

\section{Desafíos empresariales}

Encontrar tiempo para centrarse en el crecimiento de su empresa.

Acceder a la información adecuada para poder tomar decisiones

Establecer y mantener relaciones más estrechas con los clientes

Minimizar las entradas de datos duplicadas, los errores y los retrasos - Optimizar el flujo de caja para las necesidades empresariales Funciones clave

Contabilidad y finanzas: Gestione el libro mayor, los diarios, los presupuestos y la contabilidad de deudores y acreedores Ventas y gestión de relaciones con los clientes: Gestione todo el proceso de ventas, desde el primer contacto hasta el cierre de la venta y desde la gestión de los datos de clientes hasta el servicio postventa.

Compras y operaciones: Controle todo el proceso de aprovisionamiento

Inventario y distribución: Gestione el inventario en diversos almacenes y ubicaciones, efectúe un seguimiento de los movimientos de stock y regístrelos

Gestión de informes y administración: Cree, gestione y distribuya informes que mejoren el grado de transparencia y la toma de decisiones en toda la empresaBeneficios para la empresa

Más tiempo para centrarse en el crecimiento de su empresa gracias a la optimización de las operaciones• Respuestas más rápidas a las necesidades de sus clientes gracias al acceso instantáneo a información completa para actuar de forma inmediata.

Mejora de los resultados finales gracias a un único sistema integrado que elimina entradas de datos redundantes y errores, mejorando de ese modo la eficiencia de los procesos y reduciendo los costes y los retrasos 50065455 esES (10/02) @ 2010 SAP AG. Reservados todos los derechos.
Relaciones más estrechas con los clientes gracias a una información centralizada que SAP, R/3, SAP NetWeaver, Duet, PartnerEdge, ByDesign, Clear Enterprise, SAP BusinessObjects Explorer y otros productos y facilita la gestión de la comunicación con los clientes, las ventas y los contratos de servicio servicios de SAP mencionados, así como sus logotipos respectivos.

Reducción de los costes y del tiempo para la obtención de valor con una aplicación que son marcas comerciales o marcas registradas de SAP AG en Alemania y en otros países. se implementa rápidamente, es fácil de mantener y resulta lo bastante flexible para adaptarse Business Objects y el logotipo de Business Objects, BusinessOb- a las necesidades cambiantes de su empresa jects, CrystalReports, CrystalDecisions, Web Intelligence, Xcelsius y otros productos y servicios de Business Objects mencionados.

\subsection{MIE Trak Pro}

Es una solución completa de software para los fabricantes que agiliza las operaciones de negocio a través de todas las facetas de la empresa. Mediante la combinación de ventas, fabricación, ingeniería y funcionalidad de contabilidad en una sola base de datos, las empresas obtienen visibilidad en tiempo real en todos los aspectos del negocio, por lo que es más fácil de controlar los costes, gestionar el inventario y los documentos y optimizar la programación.

MIE Trak Pro ofrece a las empresas la capacidad de integrar todos los pasos, desde la cotización inicial hasta la factura final. Características del sistema incluyen MRP, trabajo que cuesta, EDI, citando, facturación, CRM, listas de materiales y códigos de barras, así como el control de inventario y los informes. Es el más adecuado para los fabricantes de encargo y los fabricantes de menos de $\$ 25$ millones en la fabricación y procesamiento de metales y puede soportar varios modos de fabricación, incluyendo discreta, MTO y ETO.

La solución ha sido diseñada para ser fácil de instalar, aprender y poner en práctica, por lo que los fabricantes pueden comenzar rápidamente a ver resultados. Además de un potente módulo citando detallada, MIE también ofrece servicios de recogida de datos Quiosco y el módulo de Programación de pizarra con la programación de arrastrar y soltar. Hay al cliente y centros de proveedores, así como una visión general de la gestión ejecutiva para garantizar que todos tengan acceso a las actualizaciones de datos en tiempo real. 
MIE Trak Pro es una excelente opción para los fabricantes que buscan una solución completamente integrada para gestionar su negocio.

Sistema operativo compatible (s): Windows XP, Windows Vista, Windows 7, Windows 2000, el navegador Web (OS agnóstico)

\subsection{MISysManufacturing}

Las empresas de fabricación de pequeñas y medianas tamaños son la base de usuarios de destino de MISysManufacturing, una solución de software completa que proporciona planificación de la producción y programación de herramientas para operaciones óptimas de compra y construcción. El objetivo final del programa es reducir la inversión en inventarios necesaria de sus usuarios, mejorar el cumplimiento de la orden y la calidad, e introducir mejores controles de costos.

Disponible como una aplicación mejor de su clase, MISys también soporta la integración con paquetes de contabilidad comunes, como SageAccpac, QuickBooks, Peachtree, y SimplyAccounting. El sistema se compone de módulos que se pueden añadir en una función de las necesidades, a partir de la fabricación de base como un componente central. Los módulos adicionales incluyen la compra avanzada, la producción avanzada, la planificación de necesidades, los controles de la planta de producción, y los módulos para el seguimiento de serie y lotes, contenedores, y el trabajo.

MISys ha estado al servicio de los fabricantes de más de un cuarto de siglo, el cultivo de relaciones estrechas con otros desarrolladores como Intuit y Sage. Productos de software Misys se venden actualmente en 48 países, con más de 7.000 clientes en todo el mundo.MISys se basa en Woodstock, Vermont. MISys recibió una calificación de rendimiento y facilidad de uso de 9,3 de cada diez de Intuit en 2010, llegando a ganar más tarde el "Grupo Sleeter impresionante Add-On" premio también.
MISysManufacturing aprovecha la tecnología de Microsoft. NET, y se basa en Microsoft SQL Express (incluido con la compra del sistema).La interfaz del sistema está construido para aprovechar la familiaridad existente de los usuarios con correo electrónico de uso común y las aplicaciones de procesamiento de textos.

\section{CONCLUSIONES}

Los beneficios derivados de la implementación de un sistema MRP varían de acuerdo a cada empresa y dependerá de la calidad del sistema antiguo en comparación con el nuevo sistema, las cuales pueden ser:

- Disminución de los stocks, las que en algunas empresas han llegado al $50 \%$.

- Mejora en el servicio al cliente que pueden incrementarse hasta un $40 \%$.

- Mejor planificación productiva.

- Menores costos y más beneficios.

- Mejor coordinación en la programación de producción e inventarios.

- Guía y ayuda en la planificación de recursos de acuerdo a la capacidad de la empresa.

\section{BIBLIOGRAFÍA}

[1] Huang, C.-Y., Huang, C.-C., \&Liu, C.-Y. (2008). Order confirmation mechanism for collaborative production networks. International Journal of Production Research, 46(3), 595-620.

[2] J.C. Anderson, R.G. Schroeder, Getting results from your MRP system, Business Horizon 27 (1984)

[3] A.D. Brown, Implementing MRP: leadership, rites andcognitive change, Logistics Information Management 7 (2) (1994). 